УДК 330.384

ББК 65.02(4Вел)

DOI 10.17150/2308-2588.2017.18(2).257-275

К. Н. Лебедев

Финансовый университет при Правительстве РФ, г. Москва, Российская Федераиия

\title{
МЕТОД ДЖ. Н. КЕЙНСА В ЭКОНОМИЧЕСКОЙ ТЕОРИИ ДЖ. М. КЕЙНСА
}

Аннотация. Качество результатов научного и практического экономического исследования в значительной мере зависит от применяемого метода исследования. K сожалению, экономические наука и практика (хозяйственное управление) вошли в XXI век с общепризнанной теорией метода исследования, весьма далекой от совершенства. Это неудивительно, так как она выступила результатом, в том числе, тех трансформаций, которые не имеют отношения к собственно науке (прежде всего идеологических и политических). В этих условиях актуальным для совершенствования теории метода экономического исследования является обращение к ее основам, разработанным на рубеже XIX-XX вв. Одним из создателей основ научного метода экономического исследования был отец Дж. М. Кейнса Джон Невилл Кейнс. При этом многие концепции экономической теории Дж. М. Кейнса являются результатами применения этого метода, прежде всего метода научной дедукции. В связи с этим сохраняющийся интерес к кейнсианской теории создает возможность для пропаганды научных начал метода экономического исследования.

Ключевые слова. Метод экономического исследования, метод научной дедукции, математический метод. 
Financial University under the Government of the Russian Federation, Moscow, Russian Federation

\section{J. N. KEYNES' METHOD IN J. M. KEYNES' THEORY}

Abstract. The method of a practical economic research determines its quality. Unfortunately, the theory of the economic practice and theory method of XXI century was not perfect. This is not surprising, as it was influenced by transformations that are not related to the actual science (like ideological and political ones). These conditions, force us to turn our mind to the bases of theoretical method of economic study, developed at the turn of XIX-XX centuries. One of the founders of the economic research scientific method was the father of J. M. Keynes John Neville Keynes and many of the concepts of the economic theory of John M. Keynes are its results. In this regard, the continued interest to the Keynesian theory creates opportunities to promote the scientific principles of the economic research method.

Keywords. Method of economic study, method of scientific deduction, mathematical method.

Упорное нежелание отечественной экономической науки возвращаться к своему научному методу, замененному при переходе к «сталинской» экономической науке на рубеже 1920-1930-х годов приукрашенной материализмом гегелевской диалектикой (уже приобретающее характер «институционной ловушки») заставляет использовать любые возможности для пропаганды научного метода экономического исследования, основы которого были разработаны к концу XIX - началу XX века. Интерес современников к кейнсианской экономической теории, наверное, предоставляет лучшую возможность для такой пропаганды, по двум причинам. Во-первых, процесс формирования одной из наиболее ярких (можно сказать «рекламных») концепций кейнсианской теории, а именно кон- 
цепции мультипликатора расходов, является не менее яркой иллюстрацией использования метода научной дедукции, составляющего основу метода экономической теории в части вывода зависимостей. Во-вторых, одним из наиболее активных создателей основ научного метода экономического исследования был отец Джона Мейнарда - Джон Невилл Кейнс ${ }^{1}$. Кроме того, анализ процесса получения именно этой концепции позволяет обратить на пользу реконструкции научного метода науки ее математизацию, а также развенчать ряд укоренившихся предрассудков, связанных с трактовкой математического метода в экономическом исследовании.

В знаменитом методологическом труде Дж. Н. Кейнса «Предмет и метод политической экономии» (1890) получают характеристику все три научных метода вывода зависимостей, а именно методы научной индукции и дедукции и математический метод. Проблемой характеристики метода научной дедукции (да и остальных методов вывода зависимостей) у Дж. Н. Кейнса, как и у других разработчиков основ научного метода экономического исследования, является, прежде всего, общая характеристика метода, смутно передающая его сущность и содержание, что, очевидно, не могло не облегчить демонтаж научного метода экономического исследования (см. выше). Как пишет Дж. Н. Кейнс, сущность дедукции «... состоит в том, что мысленно изолируются главные действующие силы и выводятся их следствия при различных условиях. На место апостериорного суждения, покоящегося всецело на исследовании конкретных фактов во всей сложности их действительного бытия, становится

1 Основы научного метода экономического исследования были разработаны в трудах таких экономистов-теоретиков, как У. С. Джевонс, Дж. Э. Кэрнс, Дж. Н. Кейнс, А. Маршалл, К. Менгер, Г. Шмоллер, В. Ф. Левитский, А. И. Чупров, А. А. Мануилов, М. И. Туган-Барановский и др. 
так[им] обр[азом] суждение априорное, основанное на предварительном знании общих начал. Такими общими началами могут быть основные психологические принципы, или уже установленные экономические законы более элементарного типа» [1, с. 162]. Описание дедукции как процесса у Дж. Н. Кейнса выглядит следующим образом: «В своем полном виде дедуктивный метод слагается из трех стадий. Необходимо, прежде всего, определить главные из действующих сил и те законы, по которым они действуют. Затем наступает чисто-дедуктивная стадия, в которой выводятся следствия, долженствующие произойти из действия этих сил при данных условиях. Наконец, путем сопоставления сделанных выводов с теми явлениями, которые доступны непосредственному наблюдению, получается возможность проверить логическую правильность и фактическую точность двух предшествующих стадий и из этой проверки извлечь указания относительно необходимых ограничений» [1, с. 162-163].

В частности, из описания дедукции как процесса видно, что Дж. Н. Кейнс в этой характеристике недалеко ушел от Дж. С. Милля, охарактеризовавшего этот метод применительно к социальному исследованию в целом полвека ранее. Вот как соответствующий исследовательский процесс описывает Дж. С. Милль в работе «Система логики силлогистической и индуктивной. Изложение принципов доказательства в связи с методами научного исследования», вышедшей в 1843 г. (для краткости ограничимся характеристикой первых двух стадий): «... Прежде всего необходимо знать... законы каждой из действующих вместе причин; это, в свою очередь, предполагает либо некоторый прежний процесс наблюдения или эксперимента над каждой причиной в отдельности, либо же прежнюю дедукцию, которая должна в своих конечных посылках зависеть от наблюдения или эксперимента» [3, с. 416]. Как пишет Дж. С. Милль далее, «когда законы причин 
установлены и удовлетворительно выполнена первая часть рассматриваемого нами теперь важного логического процесса, исследователь приступает ко второй его части: к определению (на основании найденных законов причин) того, какое именно следствие произойдет под влиянием того или другого сочетания этих причин. Это - процесс «вычисления» в очень широком смысле этого термина, весьма часто предполагающий и процессы вычисления в самом узком значении этого слова» [3, с. 420]. Таким образом, Дж. Н. Кейнс, интерпретатор метода социальных наук Дж. С. Милля применительно к экономическому исследованию, так и не раскрыл суть дедуктивного «вычисления».

Под стать общей характеристике метода научной дедукции и «примеры» его использования, которые приводит Дж. Н. Кейнс в своей книге, - он просто перечисляет исходные посылки, которыми, по его мнению, пользовались авторы соответствующих исследований, но не показывает сам процесс «вычисления» и даже его результат. Вот как Дж. Н. Кейнс описывает дедуктивное исследование «международных ценностей», осуществленное Дж. С. Миллем: «Он начинает с предположения, что международные сношения ведутся только между двумя странами и имеют объектом только два товара, которые, притом, прямо обмениваются друг на друга... Страны берутся соседние, что делает возможность не принимать в расчет издержки перевозки; обе страны свободны от всяких международных обязательств, за исключением платежа за ввозимые товары; в обоих случаях существует полная свобода торговли, - ни ввоз, ни вывоз не обложены пошлинами. Решив задачу в этой упрощенной форме, Милль отбрасывает одно за другим все поставленные ограничения, и в конце концов получает гипотезу, заключающую в себе все существенные условия современной торговли между различными человеческими обществами» [1, с. 183]. Дж. Н. Кейнс не приводит ни 2017. T. 18, № 2. C. 257-275 
процесс, ни результат решения задачи «в упрощенной форме», не говоря уже о том же самом в отношении более сложных вариантов международной торговли.

Главной причиной неясности содержания процесса дедукции в общих его характеристиках, которые приводили разработчики основ научного метода экономического исследования, является то, что метод характеризовался безотносительно к форме объекта познания, причем к такой его форме, как процесс. Этому мешало укоренившееся представление об объекте познания как о физическом теле. Таковым представлялось, прежде всего, народное хозяйство как объект политико-экономического исследования. Между тем, уже к концу XIX в., особенно в социальном исследовании, адекватной формой объекта познания, равно как и самого познания, стал процесс, а не физическое тело. Как пишут В. П. Кохановский, В. И. Пржиленский и Е. А. Сергодеева в работе «Философия науки» об изменениях, возникших на неклассическом этапе развития науки (предшествующем постнеклассическому, начавшемуся в конце XXв.), «новую трактовку получил и объект познания. Он понимается не как тело, а как процесс...» [2, с. 230]. Характеристика дедукции вне процесса как объекта ее познания, в свою очередь, не позволяла раскрыть суть дедуктивного «вычисления», состоящую в определении результатов взаимодействия «действующих сил».

Краткая характеристика дедуктивного процесса, учитывающая приведенные выше замечания, выглядит следующим образом: «Дедукция (научная) - мысленное (а также с помощью технических средств ручки, бумаги, калькулятора, ЭВМ и др.) представление изучаемого процесса как взаимодействия осуществляющего его объекта (его элементов) и его внешней среды (ее элементов) на основе знания законов их функционирования, или, по-другому, конструирование (синтез) изучаемого процесса из его элементов на 
основе знания их ситуационных вариантов и обстоятельств процесса. Дедукция, таким образом, предполагает вывод законов поведения целого на основе знания законов поведения его элементов. При этом законы поведения элементов выводятся посредством той же дедукции, научной индукции или математического метода» [4, с. 177]. Именно элементами научной дедукции выступают анализ и синтез, т. е. выделение главных причин (действующих сил) изучаемого процесса и законов их поведения и определение результатов их взаимодействия соответственно ${ }^{2}$.

Вооружившись ясным представлением о дедуктивном «вычислении», рассмотрим процесс дедуктивного вывода концепции мультипликатора расходов. Напомним ее суть: автономные изменения компонентов совокупных расходов приводят к кратным изменениям реального ВВП. В данном случае рассматриваются последствия воздействия на экономику такой меры государственной экономической политики, направленной на вывод экономики из глубокого кризиса, как организация общественных работ. Концепция мультипликатора базируется на следующих трех предпосылках, характеризующих законы поведения населения и бизнеса («действующих сил»), взаимодействие и взаимовлияние которых изучается после воздействия на экономику соответствующих мероприятий:

1. В условиях, когда в экономике имеются в значительных количествах неиспользуемые производственные мощности и незанятая рабочая сила, рост совокуп-

${ }^{2}$ Метод научной индукции представлен двумя вариантами, названными в честь сформулировавшего их Дж. С. Милля индуктивными методами разницы и аналогии. Индуктивный метод разницы - вывод, что данный процесс всегда будет иметь место при данных обстоятельствах, сделанный на основе изучения одного случая, но при полном учете всех его существенных условий и факторов. Индуктивный метод аналогии - применение для изучения нового случая зависимости, выведенной ранее при изучении другого случая, предполагающее учет всех существенных условий и факторов обоих процессов.

2017. T. 18, № 2. С. 257-275 
ного спроса не приводит к росту уровня цен, а вызывает соответствующее увеличение реального выпуска.

2. Дополнительно выпущенные в обращение деньги совершают в течение года определенное число оборотов. Оборот денег состоит в том, что полученные денежные доходы превращается в денежные расходы и выручку фирм, а выручка фирм - в подлежащие выплате доходы и далее в выплаченные доходы. В состоянии статики одна денежная единица обеспечивает объем реализации товаров и выплаты доходов в экономике, равный частоте выплаты доходов. Отсюда, полученный в конце года дополнительный доход в размере, например, 100 р., при выплате доходов раз в месяц вызовет рост совокупных расходов и номинальных доходов в следующем году на 1200 р. (100*12).

3. Наличие у людей склонности к сбережению в дополнительных доходах (предельной склонности к сбережению). Это значит, что при получении дополнительного дохода люди какую-то его часть сберегают. Эта доля измеряется коэффициентом MPS (marginal propensity to save). MPS $=\Delta S / \Delta Y$, где $S$ - сбережения, Y - доход.

Наличие предельной склонности к сбережению приводит к тому, что волна дополнительных расходов и доходов, вызванная первоначальным приростом расходов, носит затухающий характер и сходит на нет, а дополнительно выпущенные в обращение деньги постепенно изымаются из обращения и переходят в сбережения. Пусть государство в январе решило организовать дополнительные общественные работы, профинансировав их за счет кредитов центробанка (т. е. за счет выпуска дополнительных денег в обращение) на сумму 100 рублей. Безработные, получившие в январе доход в 100 р., при MPS = 0,25 потратят в феврале не 100 р., а только 75 р. $\left(100^{*}(1-0,25=100 * 0,75)\right)$. В связи с этим, в феврале выручка фирм, производящих предметы потребления, увеличится на 75 р., и на эту 
же сумму увеличатся доходы их работников, полученные в феврале. Но при том же MPS работники фирм в марте истратят на потребление не 75 р., а только 56 р. $\left(56^{*} 0,75\right)$, в связи с чем, в марте выручка фирм и доходы увеличатся только на 56 р., и т. д. В результате наличия предельной склонности к сбережению возникнет следующий ряд месячной выручки фирм и доходов: 100, 75, 56, 42, 32, 24, 18, 13, 10, 8, 6, 4, 3, 2 и т. Д. Сумма ряда равна 400 рублям. Она может быть вычислена и по формуле: $\triangle \mathrm{E} / \mathrm{MPS}$, где $\Delta \mathrm{E}-$ первоначальный прирост какого-то из компонентов совокупных расходов. И на самом деле, $100 / 0,25=400$.

Одновременно идет затухающий процесс изъятия денег из обращения в виде сбережений. В феврале будет изъято 25 р. (100-75), в марте - 19 р. (75-56), и т. д. В целом возникнет следующий ряд помесячных изъятий денег из обращения в сбережения: 25, 19, 14, 10, 8, 6, 5, 3, 2, 2, 2, 1, 1, и т. д. Его сумма равна 100 рублям.

Соединив полученный вывод с 1-й предпосылкой, получим, что первоначальный прирост какого-то из компонентов совокупных расходов вызывает кратный прирост реального ВВП, который может быть определен по формуле: $\Delta$ реал. ВВП = $\Delta \mathrm{E} / \mathrm{MPS}$. В рассмотренном примере прирост госрасходов на 100 р. вызывает прирост реального ВВП на 400 рублей.

Возможно, читатель обратил внимание на то, что в приведенном выше примере идет речь о влиянии на экономику разового автономного расхода, в то время как в концепции мультипликатора рассматривается влияние на экономику изменения уровня расходов, которому соответствовало бы ежемесячное осуществление общественных работ на сумму 100 р., т. е. ежемесячное 100-рублевое денежное «вливание» в экономику. Кроме того, фактически рассматривается процесс сокращения производства от месяца к месяцу после его первоначального «всплеска», что не соответствует «идеологии» концепции мультипликатора. 
Основываясь на картине влияния на экономику разового расхода, несложно установить, что и результатом увеличения уровня автономных расходов будет такое же кратное увеличение уровня совокупных расходов, приводящее к соответствующему увеличению уровня реального выпуска.

Если в январе дополнительные расходы составят 100 р., то в феврале - 100+75 р. (из которых 100 - февральские расходы на общественные работы, а 75 - расходы на потребление, вызванные доходами от участия в общественных работах в январе), марте - 100+75+56 (из которых 100 - мартовские расходы на общественные работы, 75 - расходы на потребление, вызванные доходами от участия в февральских общественных работах, 56 - расходы на потребление, вызванные доходами от участия в январских общественных работах), и т. д. Через некоторое количество месяцев ежемесячный уровень дополнительных расходов вплотную приблизится к 400 рублям.

В рассмотренном выше дедуктивном процессе исследования осуществлялись простейшие математические расчеты, а его результаты записывались, в том числе, в виде математических рядов. Из этого следует важный вывод: использование в процессе вывода закономерностей математических приемов обработки информации (умножение, вычитание и др.), а также математических форм отражения (таблицы, формулы, графики и др.), не является признаком математического метода. Операции с математическими символами и использование математических форм отражения могут выступать формой осуществления дедуктивных (а также индуктивных) выводов (см. ниже).

Существенной проблемой современной общепринятой теории метода экономического исследования (представленной, например, в менеджменте-науке) является то, что за основу группировки конкретных методов вывода зависимостей берется не их содержание, 
а форма, в связи с чем дедуктивные по своей природе методы благодаря математической форме зачисляются в состав количественных, или экономико-математических, методов. К математическим методам необоснованно относят, в частности, график Гантта, сетевой график, многие методы имитационного моделирования.

Заметим, что приведенные выше ряды выручки и доходов и изъятий в сбережения могли быть получены с помощью компьютера на основе простейшей программы. Из этого также следует важный вывод - и использование компьютера не является признаком математического метода.

Признаком математического метода является отрыв операций с математическими символами от содержания изучаемых процессов, т. е. от мысленных операций с ними. Так, если сложение денег, поступивших на счет фирмы в течение дня, отражает процесс их накопления, то какой реальный экономический процесс может характеризовать, например, деление прироста совокупных расходов на предельную склонность к сбережению, равное, тем не менее, приросту реального ВВП, произошедшему под влиянием этого прироста? В связи с этим использование соответствующей формулы для получения искомого результата $(\Delta$ реал. ВВП = $\triangle \mathrm{E} / \mathrm{MPS}$ ) должно трактоваться как использование математического метода.

Примером математического метода выступает известная из курса микроэкономики графическая модель «рыночное равновесие». С ее помощью последствия воздействий различных факторов рыночного спроса (доходы потребителей, цена на товары-субституты и товары-комплименты, мода на товар, общее число покупателей, ожидания покупателей и др.) и рыночного предложения (новые технологии, цены на ресурсы, налоги и дотации, ожидания продавцов, число продавцов и др.) на рынок какого-то товара (объем продаж, рыночную цену) определяются путем осу2017. T. 18, № 2. С. 257-275 
ществления право- или левосторонних сдвигов кривых рыночного спроса и предложения, соответствующих определенным факторам, и выяснения характера сдвигов проекций точки пересечения двух кривых на ось объема продаж (горизонтальную ость) и ось рыночной цены (вертикальную ось). Так, появление новой технологии изготовления товара в данной модели интерпретируется как правосторонний сдвиг кривой рыночного предложения, имеющей положительный наклон. При неподвижной кривой рыночного спроса, имеющей отрицательный наклон, проекция точки пересечения двух кривых на горизонтальную ось объема продаж сдвинется влево, что соответствует росту объема продаж, а ее проекция на вертикальную ось рыночной цены сдвинется вниз, что соответствует снижению рыночной цены. Таким образом, на основе модели рыночного равновесия можно установить, что появление новой технологии при прочих равных условиях приводит к увеличению объема продаж соответствующего товара и к снижению рыночной цены на него. В данном случае операции с графическими математическими символами (кривыми, координатными осями, перпендикулярами к ним) отрываются от содержания изучаемых экономических процессов, так как соответствующие выводы можно получать, не представляя того, что и как делается на рынке.

Важно отметить, что во многих случаях математические приемы заменяют соответствующее дедуктивное исследование. Например, выводы, получаемые с помощью модели рыночного равновесия, могут быть получены дедуктивным путем, а именно на основе знаний реакций потребителей (покупателей) и производителей (продавцов) на факторы рыночного спроса и рыночного предложения соответственно и рыночные действия друг друга. Так, рассмотренный выше вывод о влиянии на рынок какого-то товара появления новой технологии его производства может быть получен 
на основе следующих закономерностей: 1) появление новой технологии приводит к росту предложения товара (предложение - желание производителей производить и предлагать определенные количества товара при определенных ценах), в том числе величины его предложения при действующей на рынке цене, 2) избыток величины предложения товара на рынке по сравнению с величиной спроса на него по цене предыдущего равновесия вызывает конкуренцию между производителями за спрос покупателей в форме снижения цены, 3) снижение рыночной цены увеличивает величину спроса на товар со стороны потребителей, 4) снижение рыночной цены снижает величину его предложения со стороны производителей. «Сочетанием» этих «причин» и выступит вывод, полученный ранее более просто на основе графической математической модели рыночного равновесия: следствием появления новой технологии производства товара выступает увеличение объема продаж соответствующего товара (хотя и меньшее, чем первоначальный прирост его выпуска вследствие появления новой технологии) и снижение рыночной цены на него. Таким образом, графическая модель рыночного равновесия выступает непосредственной заменой соответствующих процессов научного дедуктивного исследования, облегчающей процесс исследования. Важно, однако, заметить, что использование математических приемов, как правило, исключает возможность изучения влияния на изучаемый процесс множества «второстепенных» причин, что позволяет делать дедуктивное исследование. Так, в приведенное выше дедуктивное исследование процесса мультипликации могут быть легко внесены предположения об изменении предельной склонности к сбережению, объема ежемесячных общественных работ и т. д. в течение изучаемого периода.

Дедуктивный метод, естественно, использовался в экономическом исследовании, как практическом, так 2017. T. 18, № 2. C. 257-275 
и научном, еще до его выделения разработчиками научного метода социального и экономического исследования. При этом одним из наиболее ярких примеров использования дедукции в математической форме выступает метод, который К. Маркс использовал для вывода условий реализации общественного продукта при простом и расширенном воспроизводстве, т. е. схемы простого и расширенного воспроизводства. Функционирование экономики страны представлено К. Марксом как взаимодействие двух подразделений общественного производства (производство средств производства и производство предметов потребления) и населения (капиталисты и рабочие), поведение которых подчиняется определенным законам, представленным в виде предпосылок исследования (безденежный обмен, обмен происходит в конце года и т. д.). Для анализа или определения структуры общественного продукта во 2-м году и далее используются простейшие арифметические приемы исследования (сложение, вычитание). Сами результаты исследования записаны в математической форме, в частности в виде неравенств - условия расширенного воспроизводства (например, главное условие - доходы рабочих и капиталистов I подразделения должны превышать стоимость средств производства, потребленных во II подразделении: I (v+m) > II c).

Давно назрела объективная оценка общепринятой концепции метода экономической теории, представленной в современных учебниках по экономической теории (диалектический материализм, системный подход, индукция и дедукция, анализ и синтез и др.). Ее основное содержание было сформировано на рубеже 20-30-х гг. XX века в процессе перехода к марксистско-ленинской политической экономии. Главной целью предпринятой «революционной» реконструкции научного метода экономического исследования стало «превращение» в методы вывода зависимостей 
философских законов, когда путем произвольного причисления экономических явлений к тем или иным философским категориям на основе соответствующих законов их взаимодействия можно получать «нужные» выводы. Образец такого «исследования» продемонстрировал К. Маркс при доказательстве главного положения марксистской теории - о неминуемой насильственной гибели капитализма, т. е. того, что капиталистические производственные отношения, включая отношения собственности, неизбежно, скоро и революционным путем будут заменены социалистическими (общественными) производственными отношениями, и произойдет это в силу внутриэкономических причин - вследствие развития производительных сил. Для получения необходимого доказательства К. Маркс использовал закон взаимодействия содержания и формы, взятый из гегелевской диалектики, рьяным поклонником которой он был, объявив производственные отношения формой, а производительные силы - содержанием процесса развития хозяйственного строя, и назвав увеличение масштабов производства и взаимозависимости между отдельными частями экономики усилением общественного характера производительных сил [5, с. 84]. Согласно же данному философскому закону, содержание определяет форму, приводит к ее революционной смене тогда, когда она перестает соответствовать эволюционно развивающемуся содержанию. Однако с таким же успехом, если в качестве критерия отбора на роль формы брать революционность смены, можно было бы объявить формой производительные силы, поскольку сдвиги в производительных силах происходят не менее революционным путем, чем сдвиги в производственных отношениях. Именно такими революционными сдвигами в технике и технологиях инициируются «кондратьевские» экономические циклы.

Из сказанного выше ясно, по какой причине главным методом экономического исследования стал ди2017. T. 18, № 2. C. 257-275 
алектический материализм. Но чтобы расчистить дорогу для его использования, нужно было уничтожить научный метод в части методов вывода зависимостей (научные методы индукции и дедукции, математический метод), что и было сделано, причем, с помощью той же диалектики. Индукция, дедукция, математический метод и эксперимент (форма индукции) выбыли из состава общенаучных методов экономического исследования как не имеющие диалектических пар. Впоследствии дедукция и индукция были возвращены, но получив формально-логическую трактовку, которая и сделала их парными методами ${ }^{3}$. Математический метод и эксперимент по причине «патологической» непарности оказались надолго забытыми в теории метода экономического исследования. Главным общенаучным методом был объявлен метод научной абстракции (благодаря известному утверждению К. Маркса о том, что в анализе экономических форм микроскоп и химические реактивы заменяет сила абстракции), тут же мистифицированный в диалектическом духе путем его разделения на парные методы восхождения от конкретного к абстрактному и обратного восхождения от абстрактного к конкретному. Элементы научной дедукции - анализ и синтез - также подверглись мистификации в гегелевском духе (стали трактоваться как расчленение предмета или явления на части, соединение этих частей в целое соответственно) и в значительной мере перестали представлять познавательную ценность.

Следующий этап «формирования» метода отечественной экономической теории связан с внедрением в него в конце 1960 - начале 1970-х гг. системного

${ }^{3}$ Метод индукции - вывод от частных случаев к общему, например, Иван, Петр, Степан и пр. смертны; Иван, Петр, Степан и пр. - люди; вывод: все люди смертны. Метод дедукции - вывод от общего случая к частному, например, все люди смертны; Семен - человек; вывод: Семен смертен. 
подхода Л. Берталанфи, возникшего на Западе в середине столетия. Системный подход - методология изучения организмов, требующая безоговорочного применения к изучаемым объектам или к процессу их исследования свойств организмов (целостность, наличие определенной структуры, взаимодействие между элементами, телеология, дифференциация, интеграция, саморегулирование на основе обратной связи и др.). Между тем, организмические аналогии могут помочь исследованию экономических процессов лишь на ранних стадиях их развития или исследования, а за этими границами они чреваты приписыванием неверных свойств и провалами в экономической политике. Так как аналогии с организмами в системном подходе используются путем применения к объектам исследования моделей различных систем (открытой, целенаправленной, саморегулирующейся и др.), наделенных свойствами организмов, лишь на том основании, что объект имеет свойство, вынесенное в название системы, а из названий систем их связь с органическим миром неясна, системный подход можно назвать самым агрессивным и коварным видом органицизма в экономическом исследовании.

Кроме того, системный подход отвергает научную дедукцию, выступающую главным методом вывода зависимостей в экономике, и абсолютизирует индукцию в форме эксперимента и математический метод, имеющие ограниченное применение в исследовании экономических процессов. Однако от внедрения системного подхода была и определенная польза - благодаря ему в метод экономической теории были возвращены эксперимент и математический метод.

Представленная выше история формирования общепризнанной теории метода экономико-теоретического исследования должна остановить использование традиционного приема формирования раздела о методе в учебниках по экономической теории, состоя2017. T. 18, № 2. C. 257-275 
щего в его бездумном переписывании с образцов, освященных авторитетами от науки.

\section{Єписок использованной литературы}

1. Кейнс Дж. Н. Предмет и метод политической экономии / Дж. Н. Кейнс. - М. : Тип. И.А. Баландина, 1899. - 264, $[\mathrm{IV}] \mathrm{c}$.

2. Кохановский В. П. Философия науки : учеб. пособие / В. П. Кохановский, В. И. Пржиленский, Е. А. Сергодеева. M. : МарT, 2006. - 650 с.

3. Милль Дж. С. Система логики силлогистической и индуктивной. Изложение принципов доказательства в связи с методами научного исследования / Дж. С. Милль. - М. : Изд-е Г.А. Лемана, 1914. - 880, [LXXXI] с.

4. Лебедев К. Н. Проблемы и перспективы науки «Экономический анализ» / К. Н. Лебедев. - М. : МАКС-Пресс, 2013. - 600 с.

5. Будович Ю. И. Курс отечественной политэкономии середины XIX - начала XX в. о методе экономического исследования // Вестник Финансовой академии. - 2004. - № 1. С. 78-86.

\section{Информация об авторе}

Лебедев Константин Николаевич - доктор экономических наук, доцент, департамент экономической теории, Финансовый университет при Правительстве РФ, 125993, г. Москва, Ленинградский проспект, д.49, e-mail: KNLebedev@fa.ru.

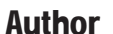

Konstantin N. Lebedev - D.Sc. in Economics, Associate Professor, Department of Economic Theory, Financial University under the Government of the Russian Federation, 49, Leningradsky Prospekt, Moscow, Russian Federation, 125993, e-mail: KNLebedev@fa.ru.

\section{Для цитирования}

Лебедев К. Н. Метод Дж. Н. Кейнса в экономической теории Дж. М. Кейнса / К. Н. Лебедев // Историко-эко- 
номические исследования. - 2017. - Т. 18, № 2. - С. 257275. - DOI: 10.17150/2308-2588.2017.18(2).257-275.

\section{7 บำ}

Lebedev K. N. J. N. Keynes' Method in J. M. Keynes' Theory. Istoriko-ekonomicheskie issledovaniya $=$ Journal of Economic History \& History of Economics, 2017, vol. 18, no. 2, pp. 257-275. DOI: 10.17150/2308-2588.2017.18(2).257-275. (In Russian). 